\title{
PD-L1 Expression and Combined Status of PD-L1/PD-1-Positive Tumor Infiltrating Mononuclear Cell Density Predict Prognosis in Glioblastoma Patients
}

\author{
Jiheun Han · Yongkil Hong ${ }^{1}$ \\ Youn Soo Lee \\ Departments of Hospital Pathology and \\ ${ }^{1}$ Neurosurgery, Seoul St. Mary's Hospital, College \\ of Medicine, The Catholic University of Korea, \\ Seoul, Korea
}

Received: June 23, 2016

Revised: August 7, 2016

Accepted: August 31, 2016

\section{Corresponding Author}

Youn Soo Lee, MD, PhD

Department of Hospital Pathology, Seoul St. Mary's

Hospital, College of Medicine, The Catholic

University of Korea, 222 Banpo-daero, Seocho-gu,

Seoul 06591, Korea

Tel: +82-2-2258-1613

Fax: +82-2-2258-1628

E-mail: lys9908@catholic.ac.kr

\begin{abstract}
Background: Programmed death ligand 1 (PD-L1) in tumor cells is known to promote immune escape of cancer by interacting with programmed cell death 1 (PD-1) in tumor infiltrating immune cells. Immunotherapy targeting these molecules is emerging as a new strategy for the treatment of glioblastoma (GBM). Understanding the relationship between the PD-L1/PD-1 axis and prognosis in GBM patients may be helpful to predict the effects of immunotherapy. Methods: PD-L1 expression and PD-1-positive tumor infiltrating mononuclear cell (PD-1+tumor infiltrating mononuclear cell [TIMC]) density were evaluated using tissue microarray containing 54 GBM cases by immunohistochemical analysis; the associations with patient clinical outcomes were evaluated. Results: PD-L1 expression and high PD-1+TIMC density were observed in $31.5 \%$ and $50 \%$ of GBM cases, respectively. High expression of PD-L1 in tumor cells was an independent and significant predictive factor for worse overall survival (OS; hazard ratio, 4.958; $p=.007$ ) but was not a significant factor in disease-free survival (DFS). PD-1+TIMC density was not correlated with OS or DFS. When patients were classified based on PD-1 expression and PD-1+TIMC density, patients with PD-L1+/PD-1+TIMC low status had the shortest OS (13 months, $p=.009)$ and DFS (7 months, $p=.053)$. Conclusions: PD-L1 expression in GBM was an independent prognostic factor for poor OS. In addition, combined status of PD-L1 expression and PD-1+TIMC density also predicted patient outcomes, suggesting that the therapeutic role of the PD-1/PD-L1 axis should be considered in the context of GBM immunity.
\end{abstract}

Key Words: Glioblastoma; Programmed cell death 1; Programmed death ligand 1
Glioblastoma (GBM) is the most common and most aggressive adult brain tumor, with a median survival of only 12 to 15 months, even with optimal treatment. ${ }^{1}$ The current management options for newly diagnosed GBM are surgical resection or biopsy, followed by radiotherapy and chemotherapy. ${ }^{2}$ Unfortunately, GBM ultimately relapses in most patients due to infiltrative growth and frequent presence of multiple lesions at the time of diagnosis. ${ }^{3}$ The limitations of conventional treatments for the improvement of GBM patient outcomes have prompted investigators to look for new therapeutic approaches and useful predictive biomarkers of treatment response. Immunotherapy, the idea of recruiting the immune system to fight against cancer, is one of these approaches.

A major determinant of cancer pathogenesis is the interaction of tumor cells with the immune system. An anticancer immune response occurs through a series of stepwise events, beginning with tumor antigen presentation by antigen-presenting cells (APCs) and progressing through priming and activation of $\mathrm{T}$ cells, trafficking of cytotoxic T cells (CD8 + cells) to tumors, and ultimately the killing of tumor cells. ${ }^{4}$ This interaction is regulated by checkpoint molecules, which can be either co-stimulatory or co-inhibitory. Programmed death ligand 1 (PD-L1) and programmed cell death 1 (PD-1) are inhibitory immune checkpoint molecules. The association between PD-1 expression on a large proportion of tumor-infiltrating lymphocytes (TILs) ${ }^{5}$ and PD-L1 upregulation in a number of cancer cells with poor clinical outcomes makes these checkpoint molecules an attractive target for an immunotherapeutic approach. ${ }^{6}$

Recent studies found that PD-L1 was overexpressed by GBM. ${ }^{7,8}$ PD-L1 on the surface of tumor cells suppresses proliferation and cytotoxic activity of T cells and promotes regulatory T-cell activity. The expression of PD-L1 in GBM is known to be regulated by both extrinsic and intrinsic mechanisms, ${ }^{9}$ and interferon $\gamma($ IFN- $\gamma$ ) mediated superinduction of PD-L1 in GBM with phosphatase and tensin homology (PTEN) deficiency has been reported. ${ }^{10}$

PD-1 is a member of the CD28 family that is expressed in 
tumor infiltrating mononuclear cells (TIMCs), including activated T cells, B cells, dendritic cells, and macrophages. ${ }^{11}$ PD-1 expression by tumor infiltrating lymphocytes (TILs) is known to be related to patient outcomes for other solid tumors. ${ }^{12-14}$

There is a current effort to develop more accurate predictive biomarkers of patient response to checkpoint blockade, particularly anti-PD-1/PD-L1, in conjunction with the tumor-host immune relationship based on PD-L1 expression and TILs. ${ }^{15,16}$ TILs, a component of the adaptive antitumor host response, are known to be related to outcomes, and adoptive transfer of TILs can mediate regression of metastatic melanoma. ${ }^{17}$

However, data on the prognostic value of PD-L1 tumor expression and PD-1 expression in tumor infiltrating immune cells in GBM are limited, and the results of previous studies have been inconsistent. In the present study, we examined PD-L1 expression and PD-1 expression in TIMCs and evaluated their prognostic value in GBM patients.

\section{MATERIALS AND METHODS}

\section{Patients}

We retrospectively identified GBM patients who were diagnosed and treated in Seoul St. Mary's Hospital, College of Medicine, The Catholic University of Korea (Seoul, Korea), between 2004 and 2012. Patient clinicopathologic and clinical follow-up data were collected from the medical records. Histologic diagnosis of glioblastoma was performed according to the current World Health Organization (WHO) classification, fourth edition. ${ }^{18}$ The ethics committee of Catholic University granted approval for this study (IRB No. KC16RISI0370).

\section{Construction of tissue microarray}

All tumor tissues were obtained at the time of the first surgery after diagnosis of GBM. A representative tumor area from each case was selected and marked on hematoxylin and eosin (H\&E)stained slides. Formalin-fixed and paraffin-embedded tissue blocks and the corresponding H\&E-stained slides were overlaid for tissue microarray (TMA) sampling. A cylindrical core (2 mm in diameter) was obtained for each case. Sectioning of microarray blocks produced $4-\mu \mathrm{m}$ thick sections.

\section{Immunohistochemistry}

Microslide tissue sections were deparaffinized with xylene, hydrated using a diluted alcohol series, and immersed in $0.3 \%$ $\mathrm{H}_{2} \mathrm{O}_{2}$ in methanol to extinguish endogenous peroxidase activity. Sections were then microwaved for 15 minutes in $10 \mathrm{mM}$ citrate buffer ( $\mathrm{pH}$ 6.0) for antigen retrieval. Each section was blocked with $4 \%$ bovine serum albumin in phosphate buffered saline with $0.1 \%$ Tween 20 (PBST) for 30 minutes to reduce non-specific staining. Sections were incubated with anti-PD-L1 (1:100, Cell Marque, Rocklin, CA, USA) or anti-PD-1 (1:100, Ventana, Tucson, AZ, USA) antibodies in PBST containing $3 \mathrm{mg} / \mathrm{mL}$ goat globulin (Sigma-Aldrich, St. Louis, MO, USA) for 60 minutes at room temperature, followed by three successive washes with a buffer. The sections were then incubated with an antimouse/rabbit antibody (Envision plus, Dako, Carpinteria, CA, USA) for 30 minutes at room temperature. The chromogen used was 3,3'-diaminobenzidine (Dako). The sections were counterstained with Meyer's hematoxylin. For positive controls, sections of human placenta and tonsil tissue were included in each staining run. Omission of the primary antibody for placenta and tonsil tissue sections was used as a negative control.

\section{Immunohistochemical analysis}

Two experienced pathologists (Y.S.L. and J.H) performed immunohistochemical analysis for PD-L1 and PD-1 by microscopic observation of the stained TMA slides. Cases were considered PD-L1 + if membranous staining of tumor cells was detected in at least $5 \%$ of the cells, irrespective of staining intensity. The cutoff threshold of PD-L1 expression was determined by receiver operating characteristic analysis of the survival rate.

PD-1 staining in TIMCs was assessed by identification of lymphocytes and macrophages on the basis of morphologic features. All TIMCs with membranous PD-1 expression of moderate to high intensity were manually counted in an entire area for each TMA core. The number of PD-1+TIMCs was divided by the total area of the TMA core $\left(3.14 \mathrm{~mm}^{2}\right)$ to obtain the number of PD-1+TIMCs per unit area $\left(/ \mathrm{mm}^{2}\right)$, the median value of which was used as a cut-off to define low versus high density.

\section{Statistical analysis}

Data were analyzed using the SPSS 21.0 statistical software package (IBM Corp., Armonk, NY, USA). Differences in patient characteristics between positive and negative PD-L1 expression or high and low density of PD-1+TIMCs were analyzed using chi-square tests, Fisher exact tests, and Student's t-tests. Comparative analysis of PD-L1 expression and the number of PD-1+ TIMCs was performed using Mann-Whitney tests. Survival time curves were drawn using the Kaplan-Meier method and a $\log$-rank test was used to assess the significance of differences in survival. Survival time included overall survival (OS; the length of time from the date of surgery or biopsy to the date of death 
from any cause, or to the last follow-up date if the patient is alive) and disease-free survival (DFS; the length of time from the date of surgery or biopsy to recurrence, progress or death from any cause). Univariate and multivariate analyses using the Cox proportional hazard model were performed to identify factors influencing OS and DFS. A step-down procedure was selected for multivariate analysis. In all statistical analyses, a two-tailed p-value less than .05 was considered to indicate a statistically significant difference.

\section{RESULTS}

\section{Patient characteristics}

The clinical characteristics of the 54 patients are summarized in Table 1. The mean patient age at diagnosis was 57.2 years, with a range of 31 to 85 years. The study group consisted of 26 males (48.1\%) and 28 females (51.9\%). Eleven patients (20.4\%) had secondary GBM that developed from anaplastic astrocytoma and oligodendroglioma. Forty patients (74.1\%) underwent total resection, $43(79.6 \%)$ were treated with adjuvant radiotherapy plus concurrent temozolomide, and nine $(16.7 \%)$ were treated with radiotherapy or temozolomide alone, based on performance status. Thirty-two patients (59.3\%) had a single lesion and 22 $(40.7 \%)$ had multifocal or multicentric lesions. Thirty-six patients (66.7\%) were alive at the last follow-up and 40 patients $(74.1 \%)$ experienced a progression or recurrence. Mean OS and DFS were 17.57 and 12.13 months, respectively.

\section{PD-L1 expression and PD-1+TIMC density in GBM}

PD-L1 staining in tumor cells was mostly of weak to moderate intensity with a membranous and cytoplasmic pattern (Fig. 1B, C). In most of the cases, PD-L1 stained only tumor cells. However,

Table 1. Summary of patient characteristics

\begin{tabular}{lc}
\hline Characteristic & No. (\%) \\
\hline Gender & \\
Male & $26(48.1)$ \\
$\quad$ Female & $28(51.9)$ \\
Primary/Secondary & \\
$\quad$ Primary & $43(79.6)$ \\
Secondary & $11(20.4)$ \\
Surgical treatment & \\
$\quad$ Total resection & $40(74.1)$ \\
Subtotal resection & $11(20.4)$ \\
Biopsy and others & $3(5.6)$ \\
Adjuvant treatment & \\
CCRT & $43(79.6)$ \\
CTx or RTx alone & $9(16.7)$ \\
No treatment & $2(3.7)$ \\
No. of lesions & \\
$\quad$ Single & $32(59.3)$ \\
$\quad$ Multiple (multifocal, multicentric) & $22(40.7)$ \\
Alive at last follow-up & \\
Yes & $36(66.7)$ \\
No & $18(33.3)$ \\
Progress/Recurrence & \\
Yes & $40(74.1)$ \\
No & $14(25.9)$ \\
Overall survival time, mean (range, mo) & $17.57(1.0-51.0)$ \\
Disease free survival, mean (range, mo) & $12.13(1.0-43.0)$ \\
\hline CCRT, concurrent chemoradiotherapy; CTx, chemotherapy; $R T x$, radiotherapy.
\end{tabular}
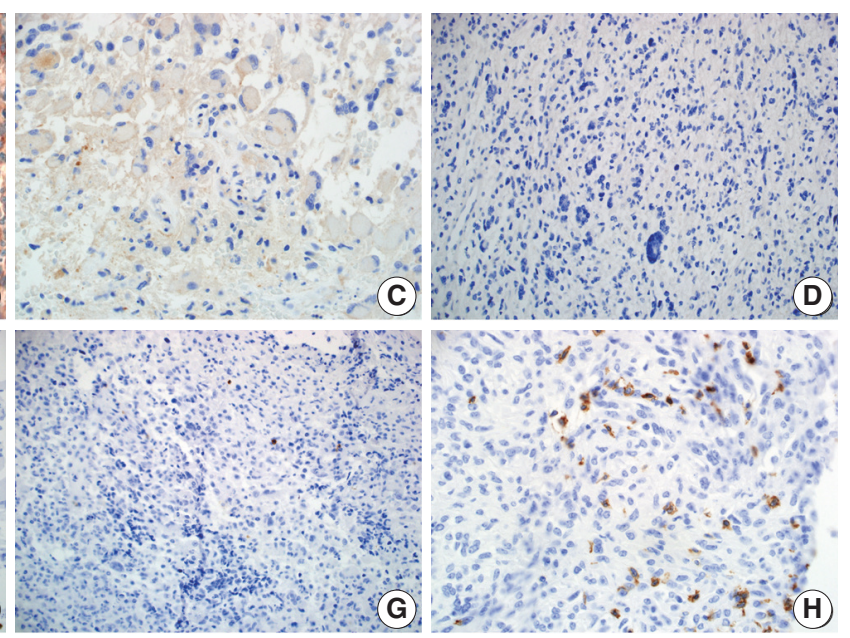

Fig. 1. Immunohistochemical staining pattern for programmed death ligand 1 (PD-L1) and programmed cell death 1 (PD-1). (A) Human placenta tissue as a positive control for endogenous PD-L1. PD-L1 positive tumor cells showing membranous and cytoplasmic staining with moderate (B) and weak (C) intensity. (D) PD-L1 negative tumor cells. (E) Tonsil tissue as a positive control for endogenous PD-1. Glioblastoma with high density $(F)$ and low density $(G)$ of PD-1 positive tumor infiltrating mononuclear cells (PD-1+tumor infiltrating mononuclear cell [TIMC]). (H) PD-1+TIMC consists of lymphocytes (most) and macrophages (some). 
in two cases (one case was PD-L1 positive, the other was not) a few dispersed TIMCs were stained with PD-L1 in their cytoplasm or membrane. In total, 17 patients $(31.5 \%)$ were positive for PD-L1 expression in tumor cells.

The median PD-1+TIMC density in GBM was $1.75 / \mathrm{mm}^{2}$ (range, 0 to $36.6 / \mathrm{mm}^{2}$ ), and 27 patients $(50 \%)$ were classified as PD-1+TIMC high density (Fig. 1F, G). Most of the PD-1+ TIMC were lymphocytes with small round condensed nuclei and rims of cytoplasm. Occasionally, the cells had elongated or kidney shaped nuclei with a scant to moderate amount of cytoplasm (which were considered to be macrophages) that were stained with PD-1 (Fig. 1H).

No significant relationship between PD-L1 expression and PD-1+TIMC density was detected.

\section{Correlations between PD-L1 expression and PD-1+TIMC density and GBM patient characteristics}

Patient characteristics and their associations with PD-L1 expression and PD-1+TIMC density are summarized in Table 2.
PD-L1 expression was significantly more frequent in patients who had died by the last follow up than in survivors ( $\mathrm{p}=.038$ ). No significant correlations were observed between PD-1+ TIMC density and patient characteristics.

\section{Prognostic implication of PD-L1 expression and PD-1+TIMC density for GBM}

Univariate analysis revealed that PD-L1 expression was significantly associated with poor OS ( $\mathrm{p}=.024$; hazard ratio [HR], 3.058; 95\% confidence interval [CI], 1.16 to 8.06) (Table 3), whereas none of the other factors, including PD-1+TIMC density, were significantly associated with OS. Multivariate analyses demonstrated that PD-L1 expression was an independent and significant predictive factor for worse OS ( $\mathrm{p}=.007$; HR, 4.958; $95 \%$ CI, 1.557 to 15.79) (Table 3).

Kaplan-Meier survival analysis revealed that patients with PD-L1 expression exhibited significantly shorter OS (median OS, 15 months vs 41 months, $\mathrm{p}=.017$ ) (Fig. 2A), but that there was no significant difference in DFS (median DFS, 10

Table 2. Relationship between PD-L1 expression and density of PD-1+TIMC and GBM patient characteristics

\begin{tabular}{|c|c|c|c|c|c|c|c|}
\hline & \multirow{2}{*}{$\begin{array}{c}\text { All cases } \\
(n=54)\end{array}$} & \multicolumn{3}{|c|}{ PD-L1 } & \multicolumn{3}{|c|}{ PD-1+TIMC } \\
\hline & & Negative & Positive & $p$-value & Low & High & $p$-value \\
\hline All cases & & $37(68.5)$ & 17 (31.5) & & $27(50)$ & $27(50)$ & \\
\hline Gender & & & & .914 & & & .586 \\
\hline Male & 26 & 18 (69.2) & $8(30.8)$ & & $14(53.8)$ & $12(46.2)$ & \\
\hline Female & 28 & 19 (67.9) & $9(32.1)$ & & $13(48.1)$ & $15(55.6)$ & \\
\hline $\begin{array}{l}\text { Age at diagnosis (yr) } \\
\text { Mean (min-max) }\end{array}$ & & $\begin{array}{c}57.62 \\
(31-85)\end{array}$ & $\begin{array}{l}56.18 \\
(36-78)\end{array}$ & .814 & $\begin{array}{c}54.26 \\
(32-77)\end{array}$ & $\begin{array}{l}60.07 \\
(31-85)\end{array}$ & .115 \\
\hline Primary/Secondary & & & & .47 & & & .311 \\
\hline Primary & 43 & $28(65.1)$ & $15(34.9)$ & & $23(53.5)$ & $20(46.5)$ & \\
\hline Secondary & 11 & $9(81.8)$ & $2(18.2)$ & & $4(36.4)$ & $7(63.6)$ & \\
\hline Surgical treatment & & & & .672 & & & .804 \\
\hline Total resection & 40 & $26(65.0)$ & $14(35.0)$ & & $19(47.5)$ & $21(52.5)$ & \\
\hline Subtotal resection & 11 & $9(81.8)$ & $2(18.2)$ & & $6(54.5)$ & $5(45.5)$ & \\
\hline Biopsy and others & 3 & $2(66.7)$ & $1(33.3)$ & & $2(66.7)$ & $1(33.3)$ & \\
\hline Adjuvant treatment & & & & .257 & & & .082 \\
\hline CCRT & 43 & 27 (62.8) & 16 (37.2) & & $23(53.5)$ & $20(46.5)$ & \\
\hline CTx or RTx alone & 9 & $8(88.9)$ & $1(11.1)$ & & $2(22.2)$ & $7(77.8)$ & \\
\hline No treatment & 2 & $2(100)$ & 0 & & $2(100)$ & 0 & \\
\hline No. of lesions & & & & .713 & & & .78 \\
\hline Single & 33 & $22(66.7)$ & $11(33.3)$ & & $16(48.5)$ & $17(51.5)$ & \\
\hline Multiple & 21 & 15 (71.4) & $6(28.6)$ & & $11(52.4)$ & $10(47.6)$ & \\
\hline Alive at last follow-up & & & & .038 & & & .248 \\
\hline Yes & 36 & 28 (77.8) & $8(22.2)$ & & $16(44.4)$ & $20(55.6)$ & \\
\hline No & 18 & $9(50.0)$ & $9(50.0)$ & & $11(61.1)$ & $7(38.9)$ & \\
\hline Progress/Recurrence & & & & 1 & & & .214 \\
\hline Yes & 40 & 27 (67.5) & $13(32.5)$ & & $22(55.0)$ & $18(45.0)$ & \\
\hline No & 14 & $10(71.4)$ & $4(28.6)$ & & $5(35.7)$ & $9(64.3)$ & \\
\hline
\end{tabular}

Values are presented as number (\%) unless otherwise indicated.

PD-L1, programmed death ligand 1; PD-1, programmed cell death 1; TIMC, tumor infiltrating mononuclear cell; GBM, glioblastoma; CCRT, concurrent chemoradiotheraphy; CTx, chemotherapy; RTx, radiation therapy. 
Table 3. Univariate and multivariate analyses of clinicopathologic factors for OS and DFS

\begin{tabular}{|c|c|c|c|c|c|c|c|c|}
\hline \multirow{3}{*}{ Variable } & \multicolumn{4}{|c|}{ OS } & \multicolumn{4}{|c|}{ DFS } \\
\hline & \multicolumn{2}{|l|}{ Univariate } & \multicolumn{2}{|l|}{ Multivariate } & \multicolumn{2}{|l|}{ Univariate } & \multicolumn{2}{|l|}{ Multivariate } \\
\hline & $\mathrm{HR}(95 \% \mathrm{Cl})$ & $p$-value & $\mathrm{HR}(95 \% \mathrm{Cl})$ & $p$-value & Hazard ratio (95\% Cl) & $p$-value & $\mathrm{HR}(95 \% \mathrm{Cl})$ & $p$-value \\
\hline \multicolumn{9}{|l|}{ PD-L1 expression } \\
\hline Positive & $3.058(1.160-8.060)$ & .024 & $4.958(1.557-15.79)$ & .007 & $1.651(0.821-3.319)$ & .16 & & \\
\hline Negative & Reference & & Reference & & Reference & & & \\
\hline \multicolumn{9}{|l|}{ PD-1+TIMC } \\
\hline High & $0.726(0.280-1.879)$ & .509 & & & $0.842(0.445-1.593)$ & .597 & & \\
\hline Low & Reference & & & & Reference & & & \\
\hline Age (continuous) & 0.989 (0.953-1.026) & .541 & & & $0.997(0.973-1.002)$ & .825 & & \\
\hline \multicolumn{9}{|l|}{ Gender } \\
\hline Male & $2.000(0.747-5.360)$ & .168 & $4.053(1.230-13.35)$ & .021 & $1.806(0.940-3.472)$ & .076 & $2.142(1.077-4.260)$ & .03 \\
\hline Female & Reference & & Reference & & Reference & & Reference & \\
\hline \multicolumn{9}{|l|}{ Primary vs secondary } \\
\hline Primary & $1.830(0.418-8.007)$ & .423 & & & $1.023(0.447-2.340)$ & .957 & & \\
\hline Secondary & Reference & & & & Reference & & & \\
\hline \multicolumn{9}{|l|}{ Numver of lesions } \\
\hline Single & Reference & .133 & Reference & .078 & Reference & .278 & Reference & .092 \\
\hline Multiple & $2.103(0.797-5.547)$ & & $2.715(0.893-8.253)$ & & 1.438 (0.746-2.772) & & $1.814(0.907-3.629)$ & \\
\hline \multicolumn{9}{|l|}{ Surgical treatment } \\
\hline Total resection & Reference & & & & Reference & & & \\
\hline Subtotal resection & $1.134(0.365-3.528)$ & .828 & & & 1.099 (0.497-2.433) & .815 & & \\
\hline Biopsy and others & $1.453(0.186-11.34)$ & .721 & & & $2.807(0.838-9.405)$ & .094 & & \\
\hline \multicolumn{9}{|l|}{ Adjuvant treatment } \\
\hline CCRT & Reference & & Reference & & Reference & & & \\
\hline CTx or RTx alone & $1.309(0.369-4.640)$ & .677 & 2.369 (0.562-9.989) & .024 & $0.706(0.245-2.030)$ & .518 & & \\
\hline No treatment & $7.717(0.880-67.674)$ & .065 & 5.760 (2.089-317.6) & .011 & $1.346(0.181-10.011)$ & .771 & & \\
\hline Recurrence or progression & $2.238(0.511-9.795)$ & .285 & & & & & & \\
\hline
\end{tabular}

OS, overall survival; DFS, disease free survival; HR, hazard ratio; Cl, confidence interval; PD-L1, programmed death ligand 1; PD-1, programmed cell death 1; TIMC, tumor infiltrating mononuclear cell; CCRT, concurrent chemoradiation therapy; CTx, chemotherapy; RTx, radiation therapy.

months vs 14 months; $\mathrm{p}=.14$ ) (Fig. 2B). There were no significant differences in OS or DFS according to PD-1+TIMC density (Fig. 2C, D).

\section{Classification of patients based on PD-L1 expression and PD-1+TIMC density}

We divided patients into four groups according to their PDL1 expression status and PD-1+TIMC density: PD-L1+/PD-1+ TIMC high (group 1; 10 patients, 18.5\%), PD-L1+/PD-1+ TIMC low (group 2; 7 patients, 13\%), PD-L1-/PD-1+TIMC high (group 3; 17 patients, 31.5\%), and PD-L1-/PD-1+TIMC low (group 4; 20 patients, 37\%). The median OS was 24 months in group 1, 13 months in group 2, 27 months in group 3, and 41 months in group $4(\mathrm{p}=.0092)$ (Fig. 3A). The median DFS was 11 months in group 1, 7 months in group 2, and 24 months in groups 3 and $4(\mathrm{p}=.053)$ (Fig. 3B). Patients in group 2 had significantly worse rates of OS (Fig. 3C, E) and DFS (Fig. 3D, F) than those in groups 3 and 4 . They also tended to have shorter OS ( $\mathrm{p}=.049)$ (Fig. 3G) and DFS ( $\mathrm{p}=.068)($ Fig. 3H) than those in group 1.

Histologically, the GBM of group 2 tended to show marked tumor cellularity, low density of TIMCs, and a high Ki-67 index $(\geq 20 \%)$. Patients in group 2 were younger at the age of diagnosis than the other groups, although this difference was not statistically significant (Table 4).

\section{DISCUSSION}

The expression rate of PD-L1 in glioblastoma patients in our study was $31.5 \%$, which is comparable to that of other solid tumors, such as $\sim 30 \%$ for melanoma ${ }^{19}$ and $\sim 36 \%$ for non-small cell lung cancer. ${ }^{20}$ In recent studies, $38.3 \%$ of $\mathrm{GBM}^{8}$ and $37.6 \%$ for newly diagnosed $\mathrm{GBM}^{7}$ had at least 5\% PD-L1 expression.

PD-L1 expression has been observed, not only in tumor cells, but also in non-cancerous interstitial cells ${ }^{21}$ and stromal lymphocytes. ${ }^{22}$ Nduom $e t$ al. ${ }^{8}$ also observed that lymphocytes consisted of nearly 30\% PD-L1 positive cells in GBM. However, in our study, PD-L1-positive lymphocytes were found in only two 

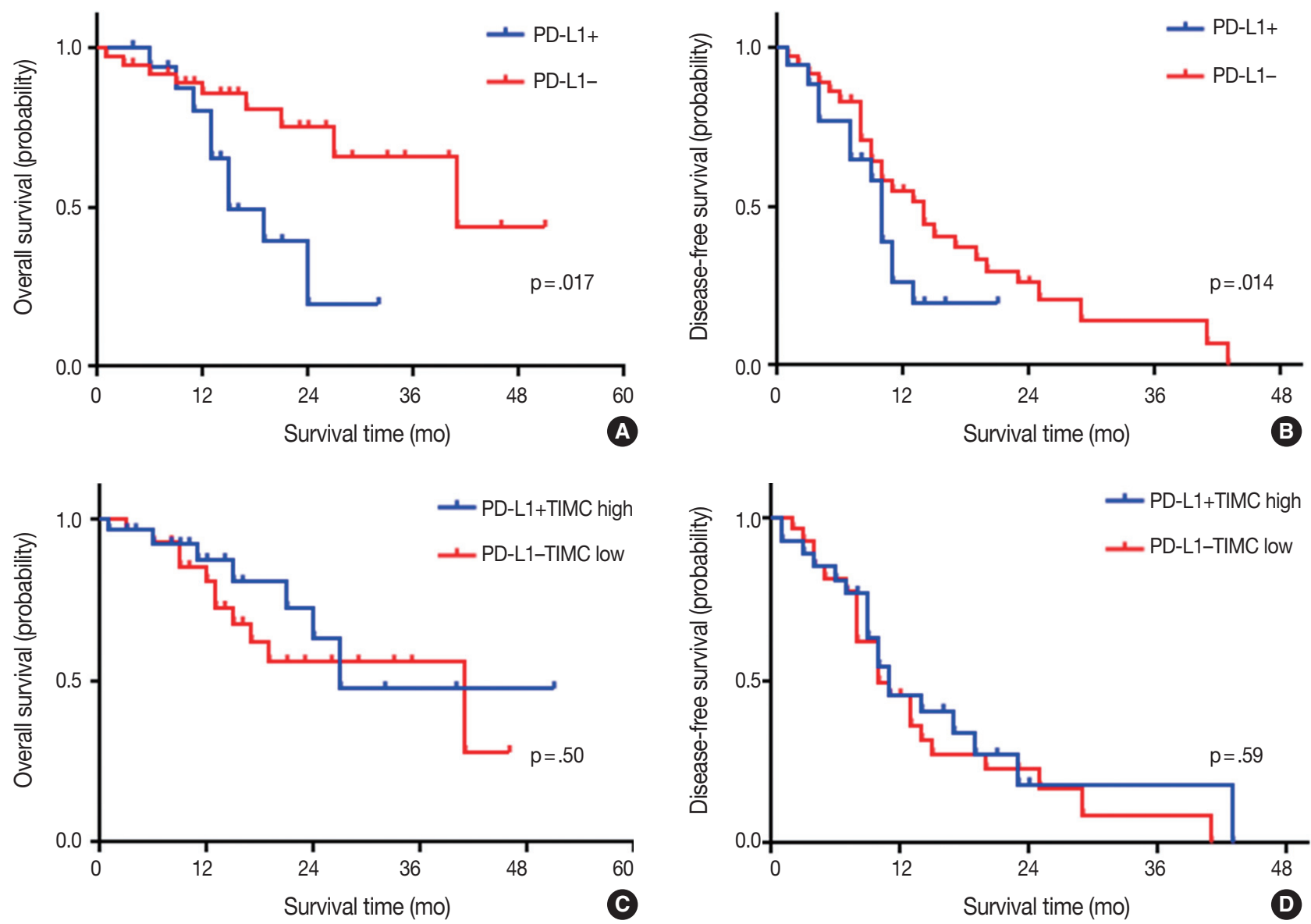

Fig. 2. Kaplan-Meier plots for overall survival and disease-free survival of glioblastoma patients according to the programmed death ligand 1 (PD-L1) expression status (A, B) and disease-free survival (C, D). TIMC, tumor infiltrating mononuclear cell.

out of 54 cases and their proportions were not significant. These differences may result from the use of different antibodies, differences in counting or detection methods, or different definitions for positivity. In addition, GBM is known to create an immunosuppressive microenvironment, resulting in sparse TILs in GBM relative to other solid tumors, which might affect the proportion of PD-L1 positive lymphocytes.

In the present study, patients with PD-L1 expression showed significantly poorer OS. However, the relationship between PDL1 expression and prognosis in patients with GBM remains unclear. Recent studies have evaluated the prognostic implications of PD-L1 expression in $\mathrm{GBM}^{7,8}$ and glioma ${ }^{23}$ with inconsistent findings. Although the precise mechanism by which intratumoral PD-L1 negatively affects patient prognosis is yet to be determined, PD-L1 has been expressed in different cancer types, including kidney, liver, ovarian, pancreatic, lung, and gastric cancer, and PD-L1 expression by tumor cells has been reported to strongly correlate with a poor prognosis. ${ }^{24-29}$

Traditionally, the central nervous system has been presumed to be an immune privileged organ, primarily due to an intact blood-brain barrier (BBB). However, in GBM, the integrity of the $\mathrm{BBB}$ is compromised, enabling activated macrophages and lymphocytes to migrate across the $\mathrm{BBB}$ into the brain parenchyma. ${ }^{30}$ Inflammatory infiltrates in GBM are relatively sparse; in the present study, the median density of PD-1+TIMCs was $1.75 / \mathrm{mm}^{2}$, which is much lower than in other solid tumors, such as lung cancer (33.4 PD-1+TILs/mm $\left.\mathrm{mm}^{2}\right){ }^{31}$ Presurgical corticosteroid treatment may affect the number of TIMCs, although Berghoff et al. ${ }^{32}$ found that corticosteroids did not affect the amount of TILs in melanoma brain metastases. It is known that GBM creates an immunosuppressive microenvironment by producing immunosuppressive cytokines, such as transforming growth factor $\beta$, prostaglandin-E, indoleamine 2,3-dioxygenase, interleukin 10, and STAT3. ${ }^{33}$ In addition, ineffective presentation of tumor antigens by APCs or recruitment of immunosuppressive cells, such as regulatory T cells (Treg) or myeloid-derived suppressor cells, is known to contribute to an immunosuppressive condition. ${ }^{33}$ The scarcity of PD- $1+$ infiltrating immune cells, 
targets of anti-PD-1 therapy, might imply reduced efficacy of the treatment.

No relationship between PD-1 expression and clinical outcomes was observed in the present study, although previous
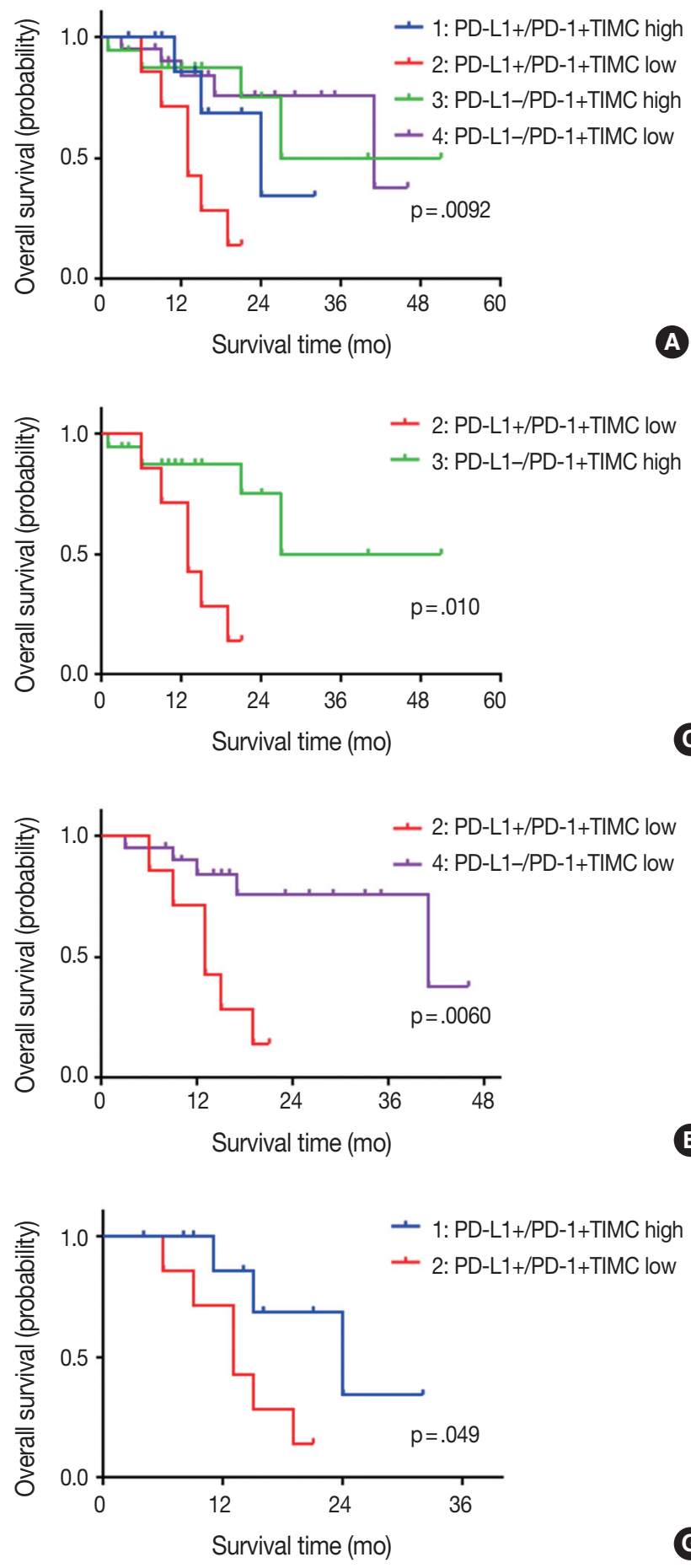

Fig. 3. Kaplan-Meier curves for overall survival (A, C, E, G), and disease free survival $(B, D, F, H)$ of patients with positive or negative expression of programmed death ligand 1 (PD-L1) and high or low density of programmed cell death 1 (PD-1)+tumor infiltrating mononuclear cell (TIMC). studies have shown inconsistent results. ${ }^{12,34}$ In a subgroup analysis dividing patients into four groups according to PD-L1 expression and PD-1+TIMC density, group 2 (PD-L1+/PD-1+TIMC low) had a significantly worse OS than the other three groups. This
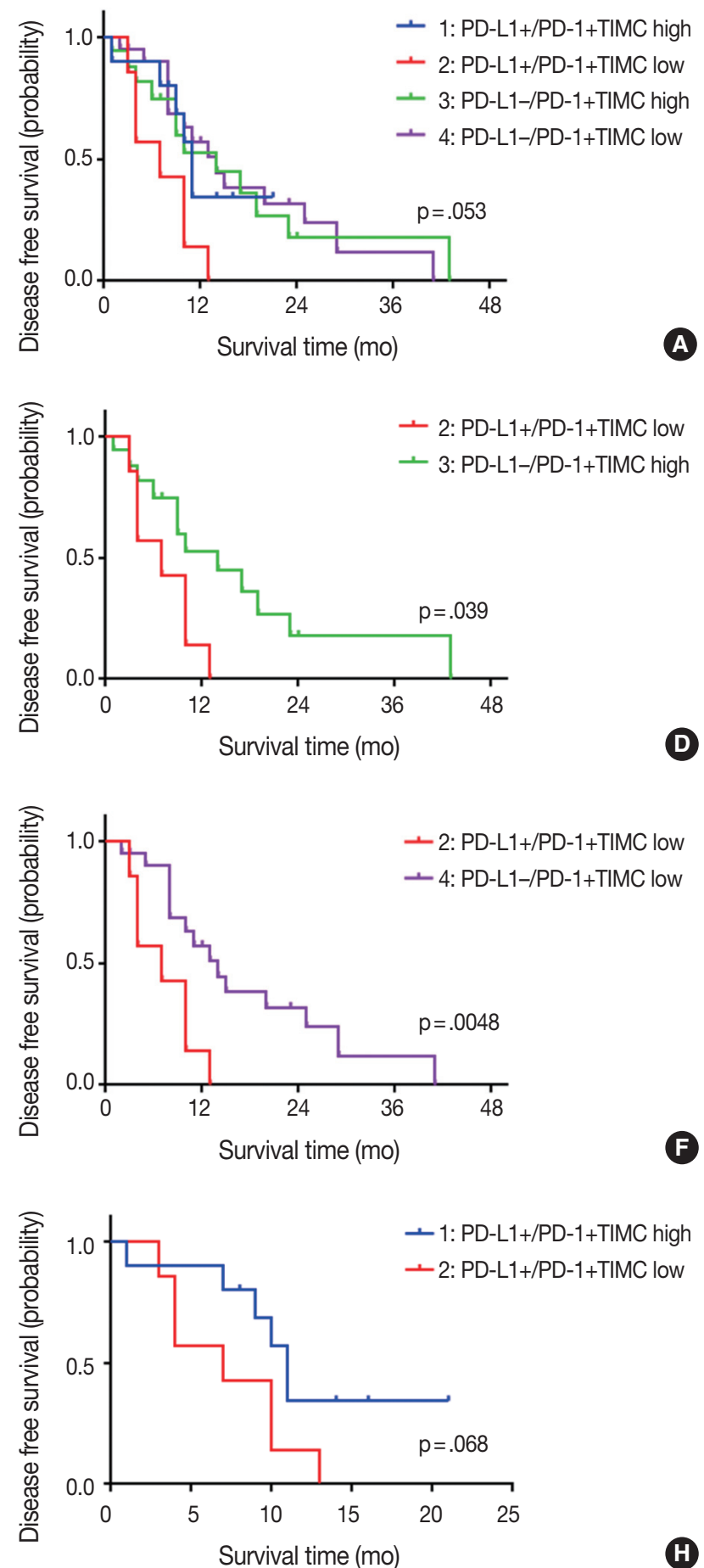
Table 4. Clinicopathological features of groups classified on the basis of PD-L1 expression and PD-1+TIMC density

\begin{tabular}{lcccc}
\hline Variable & $\begin{array}{c}\text { Group 1 } \\
\text { (PD-L1+/high PD-1+TIMC) }\end{array}$ & $\begin{array}{c}\text { Group 2 } \\
\text { (PD-L1+/low PD-1+TIMC) }\end{array}$ & $\begin{array}{c}\text { Group 3 } \\
\text { (PD-L1-/high PD-1+TIMC) }\end{array}$ & $\begin{array}{c}\text { Group 4 } \\
\text { (PD-L1-/low PD-1+TIMC) }\end{array}$ \\
\hline No. of patients (\%) & $10(18.5)$ & $7(13)$ & $17(31.5)$ & $20(37)$ \\
Age, mean (range, yr) & $59.6(40-70)$ & $51.3(36-69)$ & $60.3(31-85)$ & $55.3(32-68)$ \\
Ki-67 index, mean (range, \%) & $28.3(10-60)$ & $41.4(5-80)$ & $29.9(6-55)$ & $32.3(4-95)$ \\
Tumor cellularity & Moderate-marked & Marked & Mild-moderate & Mild-moderate \\
TIMC density & High & Low & High & Low high \\
\hline
\end{tabular}

PD-L1, programmed death ligand 1; PD-1, programmed cell death 1; TIMC, tumor infiltrating mononuclear cell.

finding was in accordance with the poor prognostic effect of PD-L1 expression. In addition, the significant difference in OS between groups 1 and 2, both of which were PD-L1 positive, and the similar clinical outcomes of groups 1, 3, and 4, suggest that the prognostic impact of PD-L1 expression on GBM should be evaluated with PD-1+TIMC density. Thus, the combined status of PD-L1 expression with PD-1+TIMC density may more precisely predict clinical outcomes.

The PD-L1 expression in GBM is mediated by IFN- $\gamma$ produced in an active immune response to the tumor or a constitutive oncogenic signaling pathway via the loss of PTEN. ${ }^{10}$ Harter et al..$^{35}$ found a loco-regional overlap between TILs and PD-L1 expression in brain metastasis, suggesting induction of PD-L1 in brain metastasis by anti-tumor immune response. However, we observed that PD-L1-positive GBM tended to have equivocal (group 1) or even lower (group 2) TIMC density than PD-L1negative groups, indicating that an intrinsic pathway might be associated with PD-L1 overexpression in GBM.

Our study has several limitations. First, the population enrolled in this study was relatively small, which could limit the statistical estimation. Second, TMA could not fully reflect the heterogeneity of GBM or the focal expression pattern of PD-L1, thus a false-negative evaluation could be possible. Lastly, as our study is retrospective, unknown factors that influence patient clinical outcomes might be present.

In summary, our study yielded two major findings: first, PDL1 expression of GBM was an independent prognostic factor associated with poor OS; second, classification of patients based on PD-L1 expression and PD-1+TIMC density also predicts patient survival. Our results suggest that PD-L1 expression might be a useful prognostic factor and should be considered in the context of PD-1+TIMCs. The combination of PD-L1 expression status and PD-1+TIMC density appears to more effectively predict patient prognosis and might be helpful for selection of appropriate candidates for immunotherapy and for evaluating immunotherapeutic efficacy.

\section{Conflicts of Interest}

No potential conflict of interest relevant to this article was reported.

\section{REFERENCES}

1. Wen PY, Kesari S. Malignant gliomas in adults. N Engl J Med 2008; 359: 492-507.

2. Weller M, van den Bent M, Hopkins K, et al. EANO guideline for the diagnosis and treatment of anaplastic gliomas and glioblastoma. Lancet Oncol 2014; 15: e395-403.

3. Thomas RP, Xu LW, Lober RM, Li G, Nagpal S. The incidence and significance of multiple lesions in glioblastoma. J Neurooncol 2013; 112: 91-7.

4. Chen DS, Mellman I. Oncology meets immunology: the cancer-immunity cycle. Immunity 2013; 39: 1-10.

5. Ahmadzadeh M, Johnson LA, Heemskerk B, et al. Tumor antigenspecific CD8 T cells infiltrating the tumor express high levels of PD-1 and are functionally impaired. Blood 2009; 114: 1537-44.

6. Okazaki T, Honjo T. PD-1 and PD-1 ligands: from discovery to clinical application. Int Immunol 2007; 19: 813-24.

7. Berghoff AS, Kiesel B, Widhalm G, et al. Programmed death ligand 1 expression and tumor-infiltrating lymphocytes in glioblastoma. Neuro Oncol 2015; 17: 1064-75.

8. Nduom EK, Wei J, Yaghi NK, et al. PD-L1 expression and prognostic impact in glioblastoma. Neuro Oncol 2016; 18: 195-205.

9. Sanmamed MF, Chen L. Inducible expression of B7-H1 (PD-L1) and its selective role in tumor site immune modulation. Cancer J 2014; 20: 256-61.

10. Han SJ, Ahn BJ, Waldron JS, et al. Gamma interferon-mediated superinduction of B7-H1 in PTEN-deficient glioblastoma: a paradoxical mechanism of immune evasion. Neuroreport 2009; 20: 1597602.

11. Keir ME, Butte MJ, Freeman GJ, Sharpe AH. PD-1 and its ligands in tolerance and immunity. Annu Rev Immunol 2008; 26: 677-704.

12. Thompson RH, Dong H, Lohse CM, et al. PD-1 is expressed by tu- 
mor-infiltrating immune cells and is associated with poor outcome for patients with renal cell carcinoma. Clin Cancer Res 2007; 13: 1757-61.

13. Muenst S, Soysal SD, Gao F, Obermann EC, Oertli D, Gillanders WE. The presence of programmed death 1 (PD-1)-positive tumorinfiltrating lymphocytes is associated with poor prognosis in human breast cancer. Breast Cancer Res Treat 2013; 139: 667-76.

14. Webb JR, Milne K, Nelson BH. PD-1 and CD103 are widely coexpressed on prognostically favorable intraepithelial CD8 T cells in human ovarian cancer. Cancer Immunol Res 2015; 3: 926-35.

15. Taube JM, Anders RA, Young GD, et al. Colocalization of inflammatory response with B7-h1 expression in human melanocytic lesions supports an adaptive resistance mechanism of immune escape. Sci Transl Med 2012; 4: 127ra37.

16. Teng MW, Ngiow SF, Ribas A, Smyth MJ. Classifying cancers based on T-cell infiltration and PD-L1. Cancer Res 2015; 75: 213945.

17. Dudley ME, Wunderlich JR, Robbins PF, et al. Cancer regression and autoimmunity in patients after clonal repopulation with antitumor lymphocytes. Science 2002; 298: 850-4.

18. Louis DN, Ohgaki H, Wiestler OD, Cavanee WK. WHO classification of tumours of the central nervous system. 4th ed. Lyon: IARC Press, 2007.

19. Weber JS, Kudchadkar RR, Yu B, et al. Safety, efficacy, and biomarkers of nivolumab with vaccine in ipilimumab-refractory or -naive melanoma. J Clin Oncol 2013; 31: 4311-8.

20. Velcheti V, Schalper KA, Carvajal DE, et al. Programmed death ligand-1 expression in non-small cell lung cancer. Lab Invest 2014; 94: 107-16.

21. Droeser RA, Hirt C, Viehl CT, et al. Clinical impact of programmed cell death ligand 1 expression in colorectal cancer. Eur J Cancer 2013; 49: 2233-42.

22. Tokito T, Azuma K, Kawahara A, et al. Predictive relevance of PDL1 expression combined with CD8+ TIL density in stage III nonsmall cell lung cancer patients receiving concurrent chemoradiotherapy. Eur J Cancer 2016; 55: 7-14.

23. Zeng J, Zhang XK, Chen HD, Zhong ZH, Wu QL, Lin SX. Expression of programmed cell death-ligand 1 and its correlation with clinical outcomes in gliomas. Oncotarget 2016; 7: 8944-55.
24. Hamanishi J, Mandai M, Iwasaki M, et al. Programmed cell death 1 ligand 1 and tumor-infiltrating CD8+ T lymphocytes are prognostic factors of human ovarian cancer. Proc Natl Acad Sci U S A 2007; 104: 3360-5.

25. Gao Q, Wang XY, Qiu SJ, et al. Overexpression of PD-L1 significantly associates with tumor aggressiveness and postoperative recurrence in human hepatocellular carcinoma. Clin Cancer Res 2009; 15: 971-9.

26. Wang L, Ma Q, Chen X, Guo K, Li J, Zhang M. Clinical significance of B7-H1 and B7-1 expressions in pancreatic carcinoma. World J Surg 2010; 34: 1059-65.

27. Thompson RH, Kuntz SM, Leibovich BC, et al. Tumor B7-H1 is associated with poor prognosis in renal cell carcinoma patients with long-term follow-up. Cancer Res 2006; 66: 3381-5.

28. Wang A, Wang HY, Liu Y, et al. The prognostic value of PD-L1 expression for non-small cell lung cancer patients: a meta-analysis. Eur J Surg Oncol 2015; 41: 450-6.

29. Liu YX, Wang XS, Wang YF, et al. Prognostic significance of PD-L1 expression in patients with gastric cancer in East Asia: a meta-analysis. Onco Targets Ther 2016; 9: 2649-54.

30. Razavi SM, Lee KE, Jin BE, Aujla PS, Gholamin S, Li G. Immune evasion strategies of glioblastoma. Front Surg 2016; 3: 11.

31. Kim MY, Koh J, Kim S, Go H, Jeon YK, Chung DH. Clinicopathological analysis of PD-L1 and PD-L2 expression in pulmonary squamous cell carcinoma: comparison with tumor-infiltrating $\mathrm{T}$ cells and the status of oncogenic drivers. Lung Cancer 2015; 88: 24-33.

32. Berghoff AS, Ricken G, Widhalm G, et al. Tumour-infiltrating lymphocytes and expression of programmed death ligand 1 (PD-L1) in melanoma brain metastases. Histopathology 2015; 66: 289-99.

33. Nduom EK, Weller M, Heimberger AB. Immunosuppressive mechanisms in glioblastoma. Neuro Oncol 2015; 17 Suppl 7: vii9vii14.

34. Duchnowska R, Peksa R, Radecka B, et al. Immune response in breast cancer brain metastases and their microenvironment: the role of the PD-1/PD-L axis. Breast Cancer Res 2016; 18: 43.

35. Harter PN, Bernatz S, Scholz A, et al. Distribution and prognostic relevance of tumor-infiltrating lymphocytes (TILs) and PD-1/PDL1 immune checkpoints in human brain metastases. Oncotarget 2015; 6: 40836-49. 